\title{
Detection of human papilloma virus and p16 expression in high-grade adenoid cystic carcinoma of the head and neck
}

\author{
Jennifer M Boland, Ellen D McPhail, Joaquín J García, Jean E Lewis and \\ David J Schembri-Wismayer
}

Department of Laboratory Medicine and Pathology, Mayo Clinic, Rochester, MN, USA

\begin{abstract}
Squamous cell carcinoma of the head and neck, particularly basaloid squamous cell carcinoma, may be difficult to distinguish from high-grade adenoid cystic carcinoma. Evidence of human papilloma virus (HPV) infection, particularly HPV 16, is frequently found in non-keratinizing oropharyngeal squamous cell carcinoma. Immunoreactivity for p16, a surrogate marker for HPV infection, often parallels the HPV infection status in oropharyngeal squamous cell carcinoma. However, the incidence and correlation between p16 expression and HPV infection in high-grade adenoid cystic carcinoma is unknown. Sixteen cases of high-grade adenoid cystic carcinoma, three cases of dedifferentiated adenoid cystic carcinoma and eight cases of low-/intermediate-grade adenoid cystic carcinoma were identified for inclusion in the study. All cases were tested by immunohistochemistry for p16 expression and in situ hybridization for high- and low-risk HPV. Eight cases (100\%) of low-to-intermediate-grade adenoid cystic carcinoma were focally positive for p16, all of which were negative for HPV. In all, 14 of 16 cases $(88 \%)$ of high-grade adenoid cystic carcinoma and three cases $(100 \%)$ of dedifferentiated adenoid cystic carcinoma were positive for p16; strong and diffuse staining was noted in three cases $(3$ of $19,16 \%)$. Two cases $(11 \%)$ of high-grade adenoid cystic carcinoma, which were also diffusely positive for p16, showed the presence of high-risk HPV. These findings suggest that the presence of HPV infection in high-grade adenoid cystic carcinoma is infrequent, even in the presence of p16 immunostaining. Nevertheless, HPV positivity should not be used to exclude the possibility of high-grade adenoid cystic carcinoma when the differential diagnosis includes squamous cell carcinoma. Moreover, although p16 overexpression is often used as a surrogate marker for HPV in squamous cell carcinoma, it cannot be used in this manner in high-grade adenoid cystic carcinoma.

Modern Pathology (2012) 25, 529-536; doi:10.1038/modpathol.2011.186; published online 9 December 2011
\end{abstract}

Keywords: adenoid cystic carcinoma; HPV; p16

Adenoid cystic carcinoma is one of the most common salivary gland tumors occurring in minor salivary gland sites, including the oropharynx. Lowto-intermediate-grade tumors typically have a classic morphological appearance, with tubular and cribriform architectural patterns predominating. ${ }^{1}$ High-grade adenoid cystic carcinoma has a predominantly solid growth pattern and a more aggressive clinical behavior. ${ }^{2}$ Dedifferentiation can also occur in adenoid cystic carcinoma, characterized by low-

Correspondence: Dr DJ Schembri-Wismayer, MD, Department of Laboratory Medicine and Pathology, Mayo Clinic, 200 First Street SW, Rochester, MN 55905, USA.

E-mail: schembriwismayer.david@mayo.edu

Received 11 May 2011; revised 4 October 2011; accepted 4 October 2011; published online 9 December 2011 or intermediate-grade tumor, juxtaposed to a poorly differentiated or undifferentiated carcinoma, and is frequently associated with poor clinical outcome. ${ }^{3}$

Due to their poorly differentiated nature and predominantly solid growth pattern, high-grade and dedifferentiated adenoid cystic carcinoma may be difficult to distinguish from non-keratinizing squamous cell carcinoma and basaloid squamous cell carcinoma, especially on small biopsy specimens. Oropharyngeal squamous cell carcinoma is frequently associated with human papilloma virus (HPV), especially HPV $16,{ }^{4-6}$ which is thought to cause p16 (cyclin-dependent kinase inhibitor 2A, $C D K N 2 A$ ) overexpression by functional inactivation of the retinoblastoma protein. ${ }^{7}$ Due to the tight correlation between p16 immunoreactivity and HPV positivity, ${ }^{6}$ p16 is widely considered a surrogate 
marker for HPV infection in the context of squamous cell carcinoma. Whether this assumption can be made in non-squamous malignancies of the head and neck is largely unknown.

Immunoreactivity for p16 has been described in adenoid cystic carcinoma of the head and neck. ${ }^{8-10}$ Although HPV positivity has not been reported in adenoid cystic carcinoma of the head and neck, it has been reported in adenoid cystic carcinoma of the uterine cervix, another frequent site of HPVassociated disease. ${ }^{11-13}$ It could therefore be hypothesized that p16 protein expression in cases of adenoid cystic carcinoma originating from the head and neck might be associated with HPV infection. The goal of this study is to test this hypothesis.

\section{Materials and methods}

The Mayo Clinic institutional review board approved the protocol for this study. The Mayo Clinic pathology database was searched for cases of adenoid cystic carcinoma of the head and neck in which resection specimens with paraffin blocks were available. This study includes one case (case 24), which was reviewed in consultation from a referring institution when the patient decided to seek care at Mayo Clinic. In this case, the existing H\&E slides, immunostains and HPV in situ hybridization (all performed at the referring institution), were reviewed. The remaining patients underwent primary surgical excision at Mayo Clinic. Existing pathology slides were reviewed, and the diagnosis of adenoid cystic carcinoma was confirmed using histomorphological criteria. Each case was graded on the basis of the predominant histological pattern: low-grade (tubular pattern), intermediate-grade (cribriform pattern) and highgrade (solid pattern). Cases with a low- or intermediate-grade adenoid cystic carcinoma, juxtaposed to a poorly differentiated carcinoma, were classified as dedifferentiated adenoid cystic carcinoma. A total of 8 cases of low- or intermediate-grade adenoid cystic carcinoma, 16 cases of high-grade adenoid cystic carcinoma and 3 cases of dedifferentiated adenoid cystic carcinoma were identified for inclusion in the study (see Table 1).

All cases were tested by immunohistochemistry for p16 expression and in situ hybridization for high- and low-risk HPV. Immunohistochemistry for p16 was performed using anti-p16 ${ }^{\text {INK4a }}$ (MTM Laboratories, Heidelberg, Germany). Staining intensity was read in a semi-quantitative fashion as follows: $0=$ no positive cells; $1+=1-25 \%$ of cells positive; $2+=26-50 \%$ of cells positive; $3+=51-75 \%$ of cells positive; $4+=76-100 \%$ of cells positive. Additional immunohistochemistry was performed on the adenoid cystic carcinoma that was positive for HPV, using antibodies against

Table 1 HPV and p16 status of 27 cases of adenoid cystic carcinoma of the head and neck

\begin{tabular}{|c|c|c|c|c|c|c|c|c|}
\hline Case & Age & Sex & Site & Diagnosis & $H P V$ & p16 & Outcome & Follow-up (months) \\
\hline 1 & 71 & M & Maxilla & IGACC & Neg & $1+$ & DOD & 48 \\
\hline 2 & 76 & $\mathrm{~F}$ & Submandibular gland & IGACC & Neg & $1+$ & DOD & 72 \\
\hline 3 & 77 & $\mathrm{M}$ & Sublingual gland & IGACC & Neg & $1+$ & DOC & 54 \\
\hline 4 & 89 & M & Soft palate & LGACC & Neg & $1+$ & DOC & 25 \\
\hline 5 & 44 & $\mathrm{M}$ & Sphenoid/nasal septum & IGACC & Neg & $2+$ & AWD & 23 \\
\hline 6 & 66 & M & Maxilla & LGACC & Neg & $2+$ & AWD & 34 \\
\hline 7 & 67 & $\mathrm{M}$ & Base of tongue & IGACC & Neg & $2+$ & ANED & 42 \\
\hline 8 & 44 & $\mathrm{M}$ & Palate & LGACC & Neg & $3+$ & ANED & 46 \\
\hline 9 & 37 & M & External auditory canal & HGACC & Neg & 0 & DOD & 26 \\
\hline 10 & 77 & $\mathrm{M}$ & Maxilla & HGACC & Neg & 0 & DOC & 27 \\
\hline 11 & 45 & $\mathrm{M}$ & Maxilla & HGACC & Neg & $1+$ & AWD & 29 \\
\hline 12 & 67 & $\mathrm{M}$ & Submandibular gland & HGACC & Neg & $1+$ & ANED & 10 \\
\hline 13 & 45 & $\mathrm{M}$ & Parotid gland & HGACC & Neg & $2+$ & Alive & 25 \\
\hline 14 & 52 & $\mathrm{~F}$ & Submandibular gland & HGACC & Neg & $2+$ & DOD & 23 \\
\hline 15 & 58 & M & Sphenoid/ethmoid & HGACC & Neg & $2+$ & DOD & 18 \\
\hline 16 & 60 & $\mathrm{M}$ & Maxilla & HGACC & Neg & $2+$ & AWD & 16 \\
\hline 17 & 64 & $\mathrm{M}$ & Lacrimal gland & HGACC & Neg & $2+$ & DOD & 17 \\
\hline 18 & 65 & $\mathrm{M}$ & Maxilla & HGACC & Neg & $2+$ & DOD & 53 \\
\hline 19 & 78 & $\mathrm{M}$ & Submandibular gland & HGACC & Neg & $2+$ & ANED & 21 \\
\hline 20 & 47 & $\mathrm{M}$ & Submandibular gland & HGACC & Neg & $3+$ & ANED & 42 \\
\hline 21 & 52 & $\mathrm{~F}$ & Parotid gland & HGACC & Neg & $3+$ & DOD & 23 \\
\hline 22 & 60 & $\mathrm{~F}$ & Larynx & HGACC & Neg & $3+$ & DOD & 44 \\
\hline 23 & 43 & $\mathrm{M}$ & Nasal cavity & HGACC & Pos & $4+$ & AWD & 27 \\
\hline 24 & 49 & $\mathrm{M}$ & Nasal cavity & HGACC & Pos & $4+$ & AWD & 35 \\
\hline 25 & 61 & $\mathrm{~F}$ & Parotid gland & DACC & Neg & $2+$ & Alive & 169 \\
\hline 26 & 56 & $\mathrm{M}$ & Parotid gland & DACC & Neg & $3+$ & ANED & 77 \\
\hline 27 & 40 & $\mathrm{M}$ & Submandibular gland & DACC & Neg & $4+$ & ANED & 6 \\
\hline
\end{tabular}

Abbreviations: ANED, alive with no evidence of disease; AWD, alive with disease; DACC, dedifferentiated adenoid cystic carcinoma; DOC, dead of other causes; DOD, dead of disease; F, female; HGACC, high-grade adenoid cystic carcinoma; HPV, human papilloma virus; IGACC, intermediate-grade adenoid cystic carcinoma; LGACC, low-grade adenoid cystic carcinoma; M, male; Neg, negative; Pos, positive. 
p63 (Biocare, Concord, CA, USA), S-100 protein (Dako, Carpinteria, CA, USA), calponin (Dako), KIT (Dako) and CK903 (Dako). In situ hybridization was performed using probes against low-risk HPV family 6 (Ventana Medical Systems, Tucson, AZ, USA; detects types 6 and 11), and separate probes against high-risk HPV family 16 (Ventana Medical Systems, detects types 16, 18, 31, 33, 35, 39, 45, 51, 52, 56, 58 and 66), all performed using the NBT/BCIP chromogen (Roche, Mannheim, Germany). In situ hybridization studies were scored as positive or negative.

Clinical histories were obtained from the existing Mayo Clinic medical record.

\section{Results}

The patients' clinical and histopathological characteristics, p16 immunohistochemistry and in situ hybridization results are summarized in Table 1 . The average patient age was 59 years (range 37-89 years). The male to female ratio was $4: 1$. Tumor locations for intermediate- and low-grade tumors included maxilla (2), submandibular gland, sublingual gland, soft palate, sphenoid sinus/nasal septum, base of tongue and palate. Sites for the high-grade adenoid cystic carcinomas included maxilla (4), submandibular gland (4), parotid gland (2), nasal cavity (2), external auditory canal, sphenoid/ ethmoid sinus, lacrimal gland and larynx. Locations of the dedifferentiated adenoid cystic carcinomas included parotid gland (2) and submandibular gland.

\section{Immunohistochemistry for p16 and In Situ Hybridization for HPV}

Both nuclear and cytoplasmic p16 positivity was seen in the tumor cells. Several patterns of heterogeneous staining were observed. Often p16-positive and p16-negative cells were intermixed within individual tumor cell nests, with approximately equal staining distribution throughout the tumor. However, in some cases, large regions of the tumor could be identified, which showed a relative increase of p16 staining compared with the surrounding tumor. Scattered p16-positive ductal and acinar cells were also seen within the background of benign salivary gland parenchyma, when present.

All eight cases $(100 \%)$ of low-to-intermediategrade adenoid cystic carcinoma were focally positive $(<75 \%$ of cells staining) for p16 (Figure 1): one case with score $3+$, three cases with score $2+$ and four cases with score $1+$. The mean score was 1.6. All the eight cases were negative for HPV by in situ hybridization.

In all, 14 of 16 cases (88\%) of high-grade adenoid cystic carcinoma were positive for p16 (Figure 2): two cases with score $4+$, three cases with score $3+$, seven cases with score $2+$ and two cases with score $1+$. The mean score was 2.1. A total of 2 of the 16 cases $(13 \%)$ of high-grade adenoid cystic carcinoma were positive for high-risk HPV family 16 by in situ hybridization (Figure 2d), both of which showed $4+$ immunopositivity for $\mathrm{p} 16$, and were located in the nasal cavity.

Three of three cases $(100 \%)$ of dedifferentiated adenoid cystic carcinoma were positive for p16 (Figure 3): one case with score $4+$, one case with score $3+$ and one with case score $2+$. The mean score was 3.0. All the three cases were negative for HPV by ISH.

\section{Additional Immunohistochemistry}

The two high-grade adenoid cystic carcinoma cases that were positive for HPV by in situ hybridization were further evaluated with immunohistochemical stains to assist in confirming the diagnosis (Figure 4). Both of these tumors showed
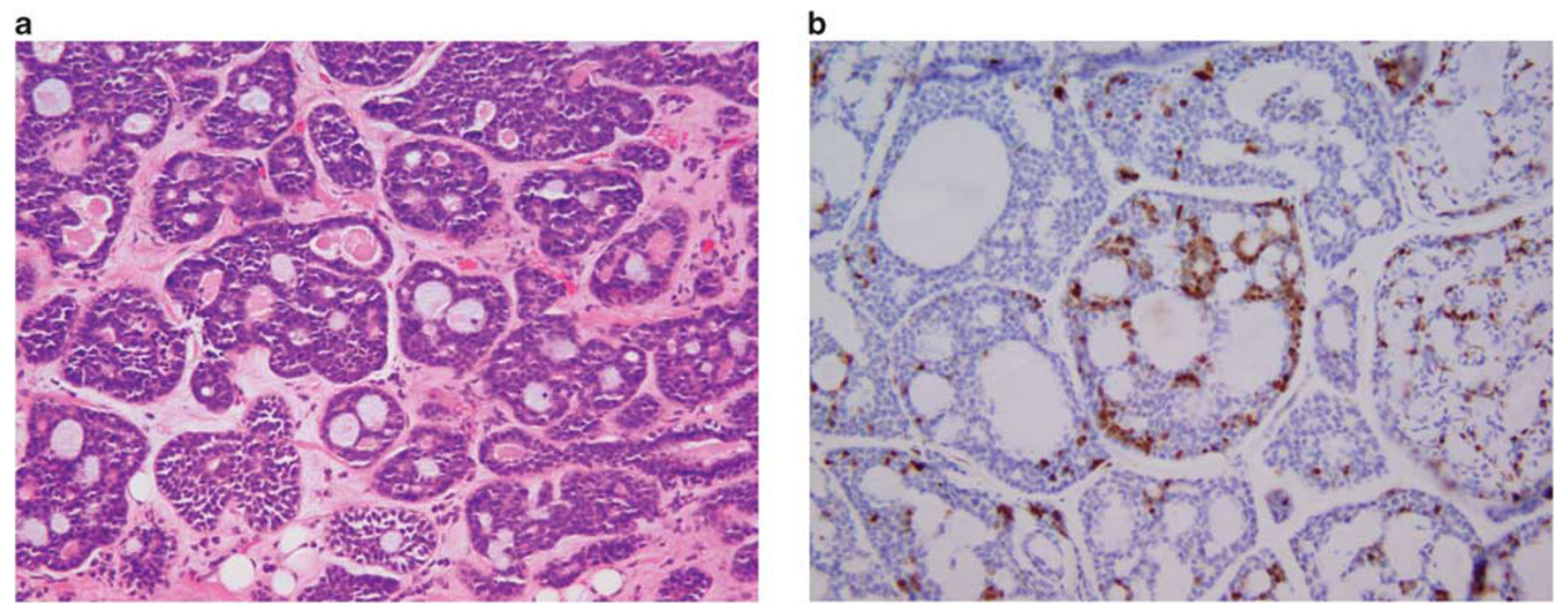

Figure 1 (a) Intermediate-grade adenoid cystic carcinoma with tubular and cribriform growth patterns. (b) p16 protein expression by immunohistochemistry, showing positivity in $1-25 \%$ of cells (score $1+$ ). HPV in situ hybridization was negative. 


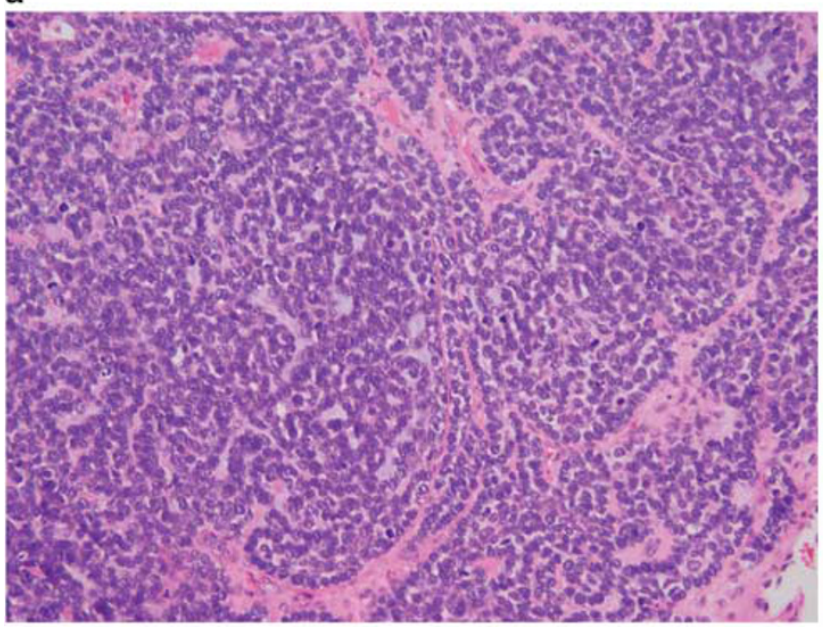

c

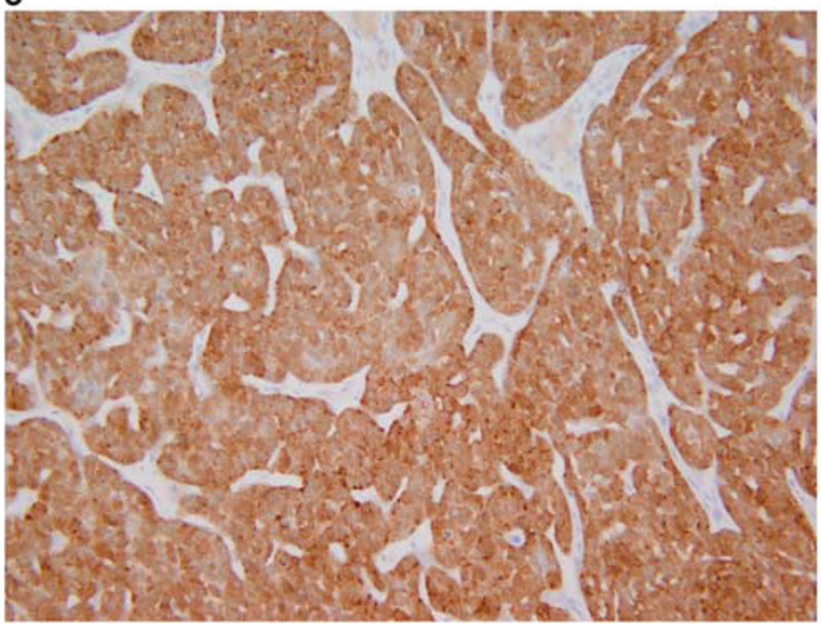

b

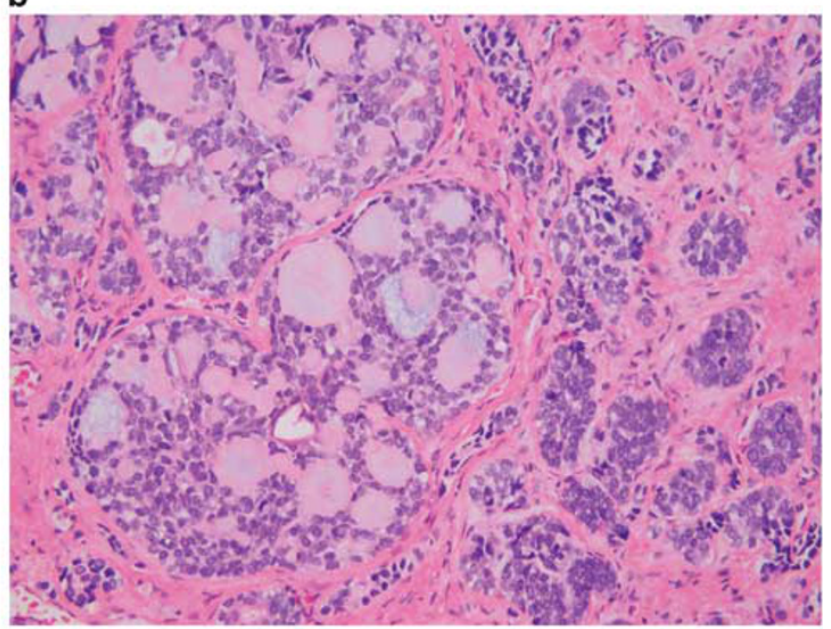

d

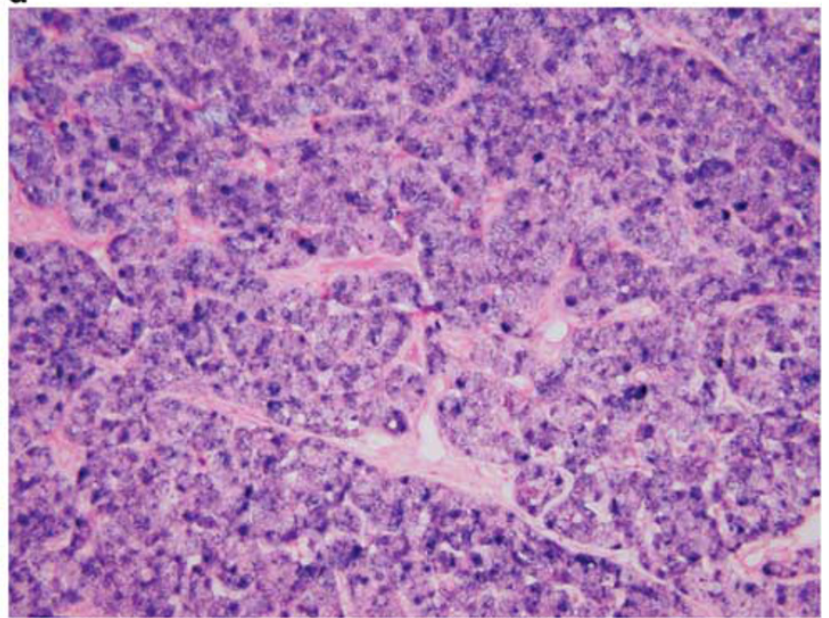

Figure 2 (a) High-grade adenoid cystic carcinoma with a predominantly solid growth pattern. (b) Other areas of the tumor show focal cribriform and tubular architecture. (c) p16 protein expression by immunohistochemistry, showing positivity in 76-100\% of cells (score $4+$ ). (d) HPV in situ hybridization is positive in the tumor cells.

a

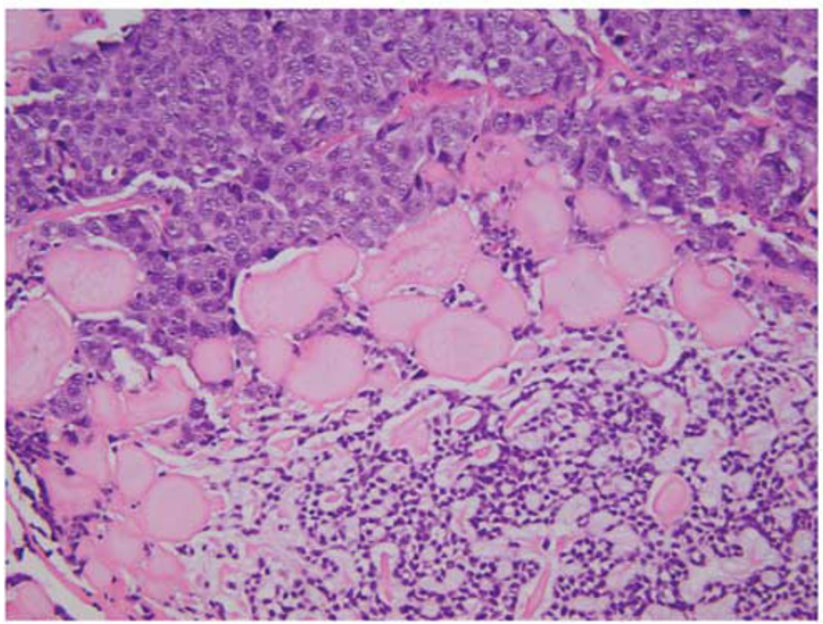

b

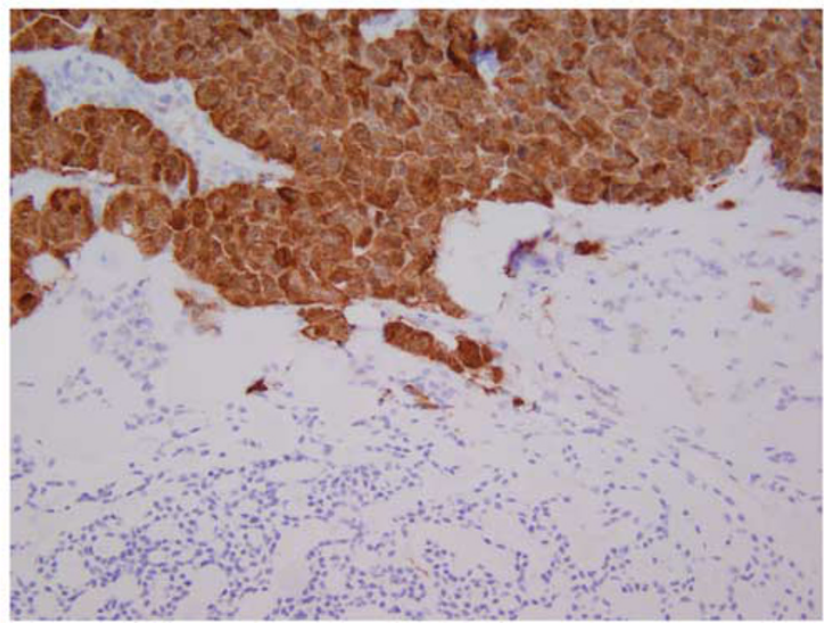

Figure 3 (a) Dedifferentiated adenoid cystic carcinoma showing a low-grade component with cribriform growth pattern, with an abrupt transition to high-grade carcinoma. (b) p16 protein expression by immunohistochemistry, showing positivity in $76-100 \%$ of cells (score $4+$ ). Note that the high-grade component shows diffuse, strong staining for p16, whereas the low-grade component shows only focal staining. HPV in situ hybridization was negative. 
a

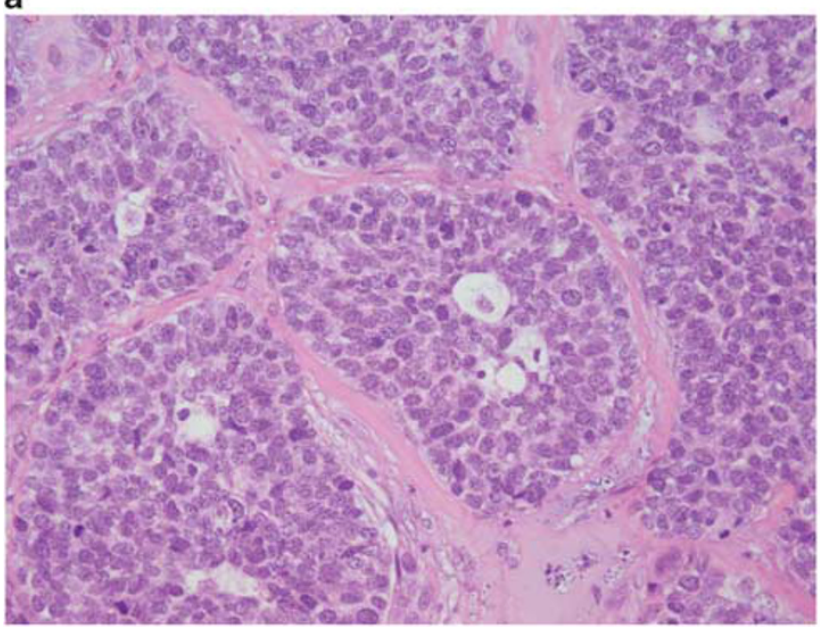

C
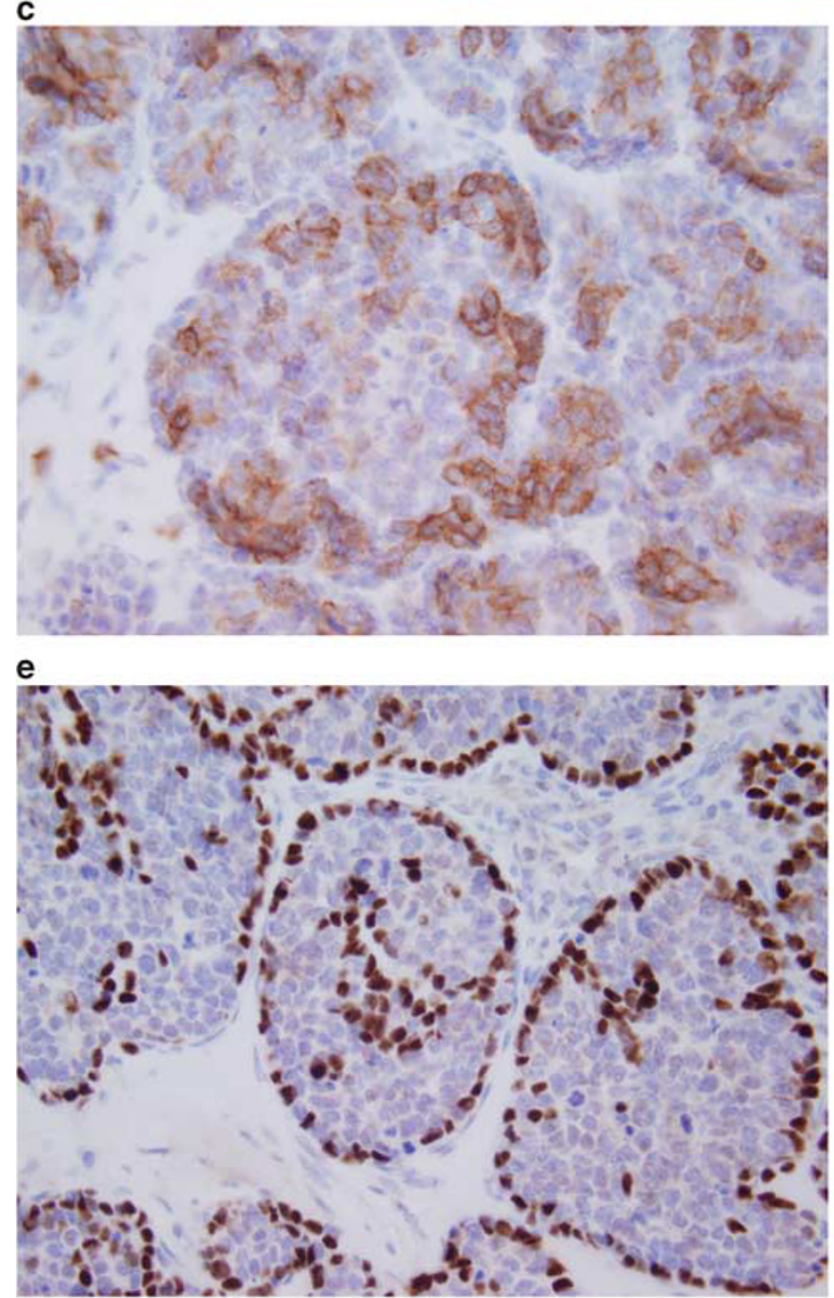

b

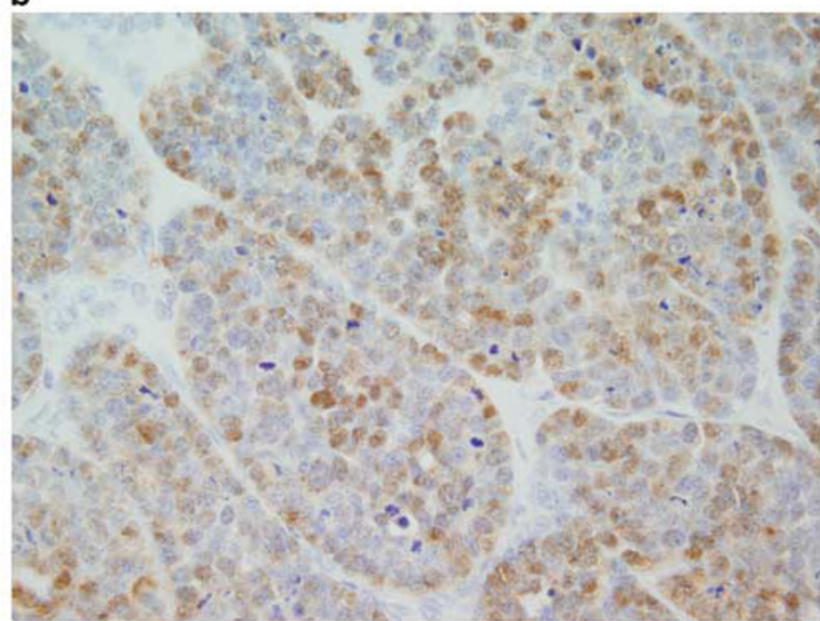

d

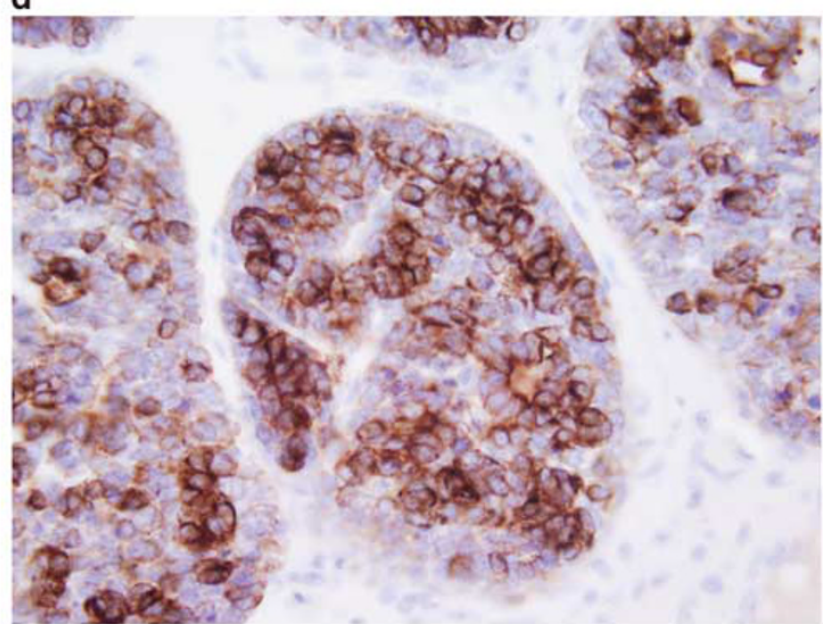

f

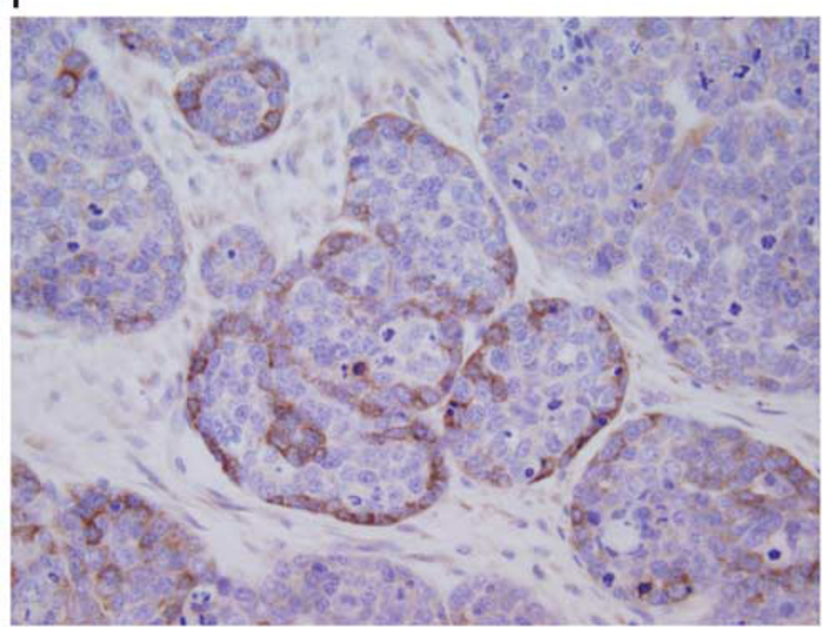

Figure 4 (a) Immunophenotype of HPV-positive high-grade adenoid cystic carcinoma (a, H\&E). Tumor cells are positive for S-100 (b), KIT (c) and keratin 903 (d), with a subset of cells staining for p63 (e; note accentuation at periphery of tumor cell nests) and calponin (f). This staining profile supports the dual ductal and myoepithelial differentiation within the tumor.

approximately $90 \%$ solid growth pattern, with $<10 \%$ of the tumors comprised by cribriform or tubular growth. The tumor cells in the center of the cell nests were positive for Keratin 903 and KIT.
Peripheral myoepithelial cells showed cytoplasmic staining with calponin and nuclear positivity for S100. Staining for p63 exhibited nuclear positivity in a subgroup of tumor cells, primarily distributed 
along the periphery of the tumor nests, but occasionally scattered throughout the tumor nests.

\section{Patient Outcome}

Clinical follow-up was available for all patients, with a mean follow-up time of 38.2 months (range 6-169 months, Table 1). Overall, nine patients (33\%) died of adenoid cystic carcinoma, three patients $(11 \%)$ died of other causes, six patients $(22 \%)$ were alive with recurrent/residual disease, seven patients $(26 \%)$ were alive with no evidence of disease, and two patients $(7 \%)$ were alive with unknown disease status.

Of the eight patients with low- or intermediategrade adenoid cystic carcinoma, two $(25 \%)$ died of disease. One patient died of locally recurrent disease 48 months after diagnosis, and one died with widely metastatic disease at 72 months after diagnosis (mean time to death, 60 months). Two patients $(25 \%)$ were alive with known locally recurrent/residual disease with 23 and 34 months of follow-up. Two patients died of other causes (25 and 54 months). Two patients were alive with no evidence of disease (42 and 46 months of follow-up).

Of the 16 patients with high-grade adenoid cystic carcinoma, 7 patients died of disease (44\%). Four patients died of locally recurrent disease at a mean of 28.5 months after diagnosis (range 17-53 months). Three patients died of widely metastatic disease, at a mean of 30 months after diagnosis (range 23-44 months). Four patients (25\%) were alive with disease, three of which had locally recurrent/ residual disease (average 24 months of follow-up, range 16-29 months) and one of which had lung metastases (35 months). One patient died of other causes (27 months). Three patients were alive with no evidence of disease (average 23.4 months of follow-up, range 10-42 months). One patient was alive with unknown disease status at 25 months.

Of the three patients with dedifferentiated adenoid cystic carcinoma, two $(67 \%)$ were alive with no evidence of disease at 6 and 77 months of follow-up. One patient (33\%) was alive with unknown disease status at 169 months.

The two patients with HPV-positive tumors were alive with known residual/recurrent disease, at the time of 27 and 35 months follow-up. One patient has known lung metastases, and the other patient has had multiple local recurrences.

\section{Discussion}

Our data show that p16 protein overexpression is frequently seen in a subset of cells in adenoid cystic carcinoma of the head and neck. This has been observed by other authors as well. ${ }^{8-10}$ It is important to note that the staining pattern is usually focal and the presence of co-existing HPV infection is uncommon, which suggests that other biological mechanisms are likely the cause of p16 overexpression in the majority of cases. Several alterations of $p 16(C D K N 2 A)$ have been described in adenoid cystic carcinoma of the head and neck, including mutations, deletions and promoter hypermethylation. ${ }^{8,9,14-16}$ p16 protein overexpression, as detected by immunohistochemistry, has also been described in adenoid cystic carcinoma of the head and neck, and in normal salivary gland tissue.$^{8-10}$ In one study, an adenoid cystic carcinoma cell line transfected with wild-type p16 (CDKN2A) showed marked decrease in proliferation activity, ${ }^{17}$ indicating that alterations in p16 $(C D K N 2 A)$ may have an important role in driving proliferation activity in some adenoid cystic carcinomas. Although a previous study by Maruya et $a l^{9}$ has described decreasing p16 protein expression with increasing grade of adenoid cystic carcinoma, we did not find this association. In our study, the degree of p16 staining seemed to be evenly distributed among high-to-low-grade cases, with a slight trend towards increasing p16 protein expression with increasing grade.

In contrast to the focal $(<75 \%)$ staining seen in most of our cases, three cases (one dedifferentiated and two high-grade adenoid cystic carcinomas) showed strong and diffuse staining for p16. The two cases of high-grade adenoid cystic carcinoma showing this staining pattern were positive for highrisk HPV 16 family by in situ hybridization. Both cases were reviewed carefully and did exhibit characteristic morphological features of adenoid cystic carcinoma, with approximately $90 \%$ solid growth and only $10 \%$ cribriform and tubular architecture. However, additional immunohistochemical studies were also performed on these two cases to ensure that these tumors were truly high-grade adenoid cystic carcinomas and not basaloid squamous cell carcinomas that were morphologically mimicking high-grade adenoid cystic carcinoma. High-grade adenoid cystic carcinoma and basaloid squamous cell carcinoma may exhibit overlapping morphological features, but usually this differential diagnosis may be resolved with careful histological evaluation. Additional sampling may be required to identify the characteristic findings of adenoid cystic or basaloid squamous cell carcinoma, which may be focal. Basaloid squamous cell carcinoma is a specific subtype of squamous cell carcinoma, defined by two main histological components. The first is a basaloid component, comprised of hyperchromatic cells with scant cytoplasm, growing in solid nests with a lobular configuration. ${ }^{1,18}$ The tumor characteristically has small spaces filled with myxoid material and stromal hyalinization. Comedo-type necrosis is common, and the cell nests may show peripheral palisading. The second component is squamous differentiation, which can be seen focally within the tumor, or may represent a larger component that is admixed with the basaloid areas. ${ }^{1,18}$ In some cases, the squamous component may be represented by only in situ squamous cell carcinoma at the mucosal 
surface. The basaloid appearance and spaces filled with myxoid material may lead to a close resemblance to adenoid cystic carcinoma. However, adenoid cystic carcinoma usually lacks squamous differentiation. The most important differentiating characteristic of adenoid cystic carcinoma is the presence of a myoepitheial cell component. ${ }^{1}$

Immunohistochemistry may be helpful in differentiation of adenoid cystic from basaloid squamous cell carcinoma, but there is also significant immunophenotypic overlap. ${ }^{19}$ In the two cases in question, our stains revealed a dual cell population, with a luminal (ductal) and myoepithelial (basal) component, which is typical of adenoid cystic carcinoma. The myoepithelial component stained with calponin and S100. p63 has also been suggested by other authors to be valuable in differentiating adenoid cystic from basaloid squamous cell carcinoma. Although both tumors are positive, basaloid squamous cell carcinomas typically show strong and diffuse positivity throughout, whereas adenoid cystic carcinomas typically show positivity in only a subset of cells, frequently located at the periphery of the tumor cells nests, or sometimes within the tumor cell nests mixed with a p63-negative cell population. ${ }^{20,21}$ This characteristic pattern of p63 positivity was seen in our two cases of high-grade adenoid cystic carcinoma that were positive for HPV, supporting our morphological impression. Both of these cases occurred in the nasal cavity of young to middle-aged men (aged 43 and 49 years). Both patients are experiencing progressive disease as would be expected for high-grade adenoid cystic carcinoma, one with lung metastases and the other with locally recurrent disease. The occurrence of distant (lung) metastases in a patient with no known nodal disease is also more in keeping with the natural history of high-grade adenoid cystic carcinoma than basaloid squamous cell carcinoma, which typically present with bulky nodal disease before the development of distant metastases. ${ }^{1}$

Although p16 protein expression has been described in adenoid cystic carcinoma of the head and neck, associated HPV infection has not been previously reported. However, adenoid cystic carcinoma of the uterine cervix has been associated with other HPV-related lesions, ${ }^{13}$ and high-risk HPV types 16, 31 and 33 have been detected in some of these tumors. ${ }^{11,12}$ Other mechanisms of p16 overexpression appear to be more common in ACC, but it is important to note that rare cases may be associated with HPV, especially when p16 staining is strong and diffuse. This finding may lead to further confusion when diagnostic considerations include non-keratinizing squamous cell carcinoma. Therefore, it is important to keep in mind that the presence of HPV should not be used to exclude the possibility of high-grade adenoid cystic carcinoma when the differential diagnosis includes both nonkeratinizing squamous cell carcinoma and highgrade adenoid cystic carcinoma.
Although the role of HPV infection and p16 overexpression in non-keratinizing squamous cell carcinoma of the oropharynx is well established, the role of this pathogenic mechanism in basaloid squamous cell carcinoma, the closest morphological mimic of high-grade adenoid cystic carcinoma, is controversial. Some studies have shown no association of HPV/p16 expression with basaloid squamous cell carcinoma, whereas other recent studies have suggested that HPV may be present and accompanied by strong p16 expression, especially in basaloid squamous cell carcinoma of the oropharynx. ${ }^{22-}$ ${ }^{25}$ It is also important to keep in mind that strong and diffuse p16 staining can occur in adenoid cystic carcinoma, even in the absence of co-existing HPV infection, and focal p16 expression is extremely common in these tumors. Lastly, although p16 overexpression is often used as a surrogate marker for HPV infection in squamous cell carcinoma, it cannot be used in this manner in high-grade adenoid cystic carcinoma.

The sample size in our study is too small to determine if there is any significant difference in the outcome of HPV-positive high-grade adenoid cystic carcinoma as compared with the more common HPV-negative tumors. On the basis of our limited data, high-grade adenoid cystic carcinomas seemed to have a higher rate of adenoid cystic-related death $(44 \%)$ when compared with low- and intermediategrade tumors $(25 \%)$, and the interval between diagnosis and adenoid cystic-related death was shorter (29 vs 60 months). The recurrence/residual disease rate was $25 \%$ in both low-/intermediategrade and high-grade adenoid cystic carcinoma. Our group of dedifferentiated adenoid cystic carcinoma was too small to make predictions about outcome, but all three patients were alive at last follow-up.

In summary, p16 overexpression is commonly observed in adenoid cystic carcinoma of the head and neck. This staining is usually focal and presumably caused by non-HPV-related mechanisms of protein overexpression. In the minority of cases where p16 staining is diffuse and strong, it is sometimes (but not always) associated with co-existing HPV infection. These findings are especially important to consider in cases where the differential diagnosis includes both adenoid cystic and squamous cell carcinoma. In this scenario, HPV and p16 positivity cannot be used to rule out the diagnosis of adenoid cystic carcinoma.

\section{Disclosure/conflict of interest}

The authors declare no conflict of interest.

\section{References}

1 Barnes L, Eveson JW, Reichart P, et al. (eds). Pathology \& Genetics of Head and Neck Tumors. World Health 
Organization Classification of Tumours, chapter 3 pages 124-126 and chapter 5 pages 221-223. IARC Press: Lyon, France, pp 430.

2 Matsuba HM, Spector GJ, Thawley SE, et al. Adenoid cystic salivary gland carcinoma. A histopathologic review of treatment failure patterns. Cancer 1986; 57:519-524.

3 Cheuk W, Chan JK, Ngan RK. Dedifferentiation in adenoid cystic carcinoma of salivary gland: an uncommon complication associated with an accelerated clinical course. Am J Surg Pathol 1999;23:465-472.

4 El-Mofty SK, Lu DW. Prevalence of human papillomavirus type 16 DNA in squamous cell carcinoma of the palatine tonsil, and not the oral cavity, in young patients: a distinct clinicopathologic and molecular disease entity. Am J Surg Pathol 2003;27:1463-1470.

5 Syrjanen S. Human papillomavirus (HPV) in head and neck cancer. J Clin Virol 2005;32(Suppl 1):59-66.

6 Singhi AD, Westra WH. Comparison of human papillomavirus in situ hybridization and p16 immunohistochemistry in the detection of human papillomavirus-associated head and neck cancer based on a prospective clinical experience. Cancer 2010;116: 2166-2173.

7 Lukas J, Parry D, Aagaard L, et al. Retinoblastomaprotein-dependent cell-cycle inhibition by the tumour suppressor p16. Nature 1995;375:503-506.

8 Nishimine M, Nakamura M, Kishi M, et al. Alterations of $p 14 A R F$ and $p 16 I N K 4 a$ genes in salivary gland carcinomas. Oncol Rep 2003;10:555-560.

9 Maruya S, Kurotaki H, Shimoyama N, et al. Expression of p16 protein and hypermethylation status of its promoter gene in adenoid cystic carcinoma of the head and neck. ORL J Otorhinolaryngol Relat Spec 2003;65:26-32.

10 Etges A, Nunes FD, Ribeiro KC, et al. Immunohistochemical expression of retinoblastoma pathway proteins in normal salivary glands and in salivary gland tumours. Oral Oncol 2004;40:326-331.

11 Daponte A, Grayson W, Moisuc D, et al. Adenoid cystic carcinoma stage Ib1 treated with radical surgery displaying human papilloma virus 33 (HPV 33): immunoelectron microscopy and review. Gynecol Oncol 2003;90:673-676.

12 Grayson W, Taylor L, Cooper K. Detection of integrated high risk human papillomavirus in adenoid cystic carcinoma of the uterine cervix. J Clin Pathol 1996;49: 805-809.

13 Yang YJ, Gordon GB. Cervical adenoid cystic carcinoma coexisting with multiple human papillomavirus- associated genital lesions. A common etiology? Gynecol Obstet Invest 1999;47:272-277.

14 Suzuki N, Onda T, Yamamoto N, et al. Mutation of the p16/CDKN2 gene and loss of heterozygosity in malignant mucosal melanoma and adenoid cystic carcinoma of the head and neck. Int J Oncol 2007;31:1061-1067.

$15 \mathrm{Li} \mathrm{J}$, El-Naggar A, Mao L. Promoter methylation of p16INK4a, RASSF1A, and DAPK is frequent in salivary adenoid cystic carcinoma. Cancer 2005;104:771-776.

16 Cerilli LA, Swartzbaugh JR, Saadut R, et al. Analysis of chromosome 9p21 deletion and p16 gene mutation in salivary gland carcinomas. Hum Pathol 1999;30: 1242-1246.

17 Zhang Y, Liu J, Xue F, et al. p16 tumor suppressor therapy in salivary adenoid cystic carcinoma cell line SACC83. Oral Surg Oral Med Oral Pathol Oral Radiol Endod 2005;100:70-74.

18 Wain SL, Kier R, Vollmer RT, et al. Basaloid-squamous carcinoma of the tongue, hypopharynx, and larynx: report of 10 cases. Hum Pathol 1986;17:1158-1166.

19 Morice WG, Ferreiro JA. Distinction of basaloid squamous cell carcinoma from adenoid cystic and small cell undifferentiated carcinoma by immunohistochemistry. Hum Pathol 1998;29:609-612.

20 Emanuel $\mathrm{P}$, Wang $\mathrm{B}$, Wu $\mathrm{M}$, et al. p63 Immunohistochemistry in the distinction of adenoid cystic carcinoma from basaloid squamous cell carcinoma. Mod Pathol 2005;18:645-650.

21 Serrano MF, El-Mofty SK, Gnepp DR, et al. Utility of high molecular weight cytokeratins, but not p63, in the differential diagnosis of neuroendocrine and basaloid carcinomas of the head and neck. Hum Pathol 2008;39: 591-598.

22 Chernock RD, Lewis Jr JS, Zhang Q, et al. Human papillomavirus-positive basaloid squamous cell carcinomas of the upper aerodigestive tract: a distinct clinicopathologic and molecular subtype of basaloid squamous cell carcinoma. Hum Pathol 2010;41: 1016-1023.

23 Begum S, Westra WH. Basaloid squamous cell carcinoma of the head and neck is a mixed variant that can be further resolved by HPV status. Am J Surg Pathol 2008;32:1044-1050.

24 Cabanillas R, Rodrigo JP, Ferlito A, et al. Is there an epidemiological link between human papillomavirus DNA and basaloid squamous cell carcinoma of the pharynx? Oral Oncol 2007;43:327-332.

25 Kim JY, Cho KJ, Lee SS, et al. Clinicopathologic study of basaloid squamous carcinoma of the upper aerodigestive tract. J Korean Med Sci 1998;13:269-274. 